\title{
An audit of thyroid function testing in acutely ill patients at a South African academic hospital
}

\author{
E C Kruger, ${ }^{1,2}$ MB ChB; OrcID 0000-0002-0312-5189; M Conradie, ${ }^{2}$ MB ChB, PhD; OrcID 0000-0003-3092-4098; \\ A Coetzee, ${ }^{2} \mathrm{MB}$ ChB, MMed (Int), FCP (SA), FC Endo (SA), MPhil; OrcID 0000-0001-9993-6439; \\ M Hoffmann, ${ }^{1,2}$ MB ChB, MMed; OrcID 0000-0002-1736-755X \\ ${ }^{1}$ National Health Laboratory Service (NHLS), Cape Town, South Africa \\ ${ }^{2}$ Department of Medicine, Faculty of Health Sciences, Stellenbosch University, Tygerberg Hospital, Cape Town, South Africa
}

Corresponding author: E C Kruger (elsie.kruger@nhls.ac.za)

\begin{abstract}
Background. Non-thyroidal illness syndrome (NTIS) can be defined as the presence of abnormal thyroid function in the absence of primary thyroid dysfunction and is associated with acute illness. Thyroid function testing is generally not advised in the critically ill patient. Thyroidstimulating hormone (TSH) measurement is the preferred screening test for thyroid disease, but results may be misleading and variable in the setting of critical illness.

Objective. To describe the pattern of requests for TSH testing in patients admitted to the emergency department and intensive care units at Tygerberg Hospital, Cape Town, South Africa.

Methods. A retrospective, descriptive (observational) study was conducted over a 6-month period to determine the number of requests for TSH testing received for patients admitted to the emergency department and intensive care units.

Results. A total of 1139 requests for TSH testing were received from the emergency department and intensive care units, of which 166 were excluded. Of the 973 requests evaluated, 14\% yielded abnormal results. The majority (79.4\%) of the abnormal TSH results were most likely attributed to NTIS. Follow-up TSH results after 6 - 8 weeks were available in only $18 \%$ of cases with abnormal TSH levels at initial presentation. Conclusion. We found that TSH testing was often requested in critically ill patients, but that most results were either normal or indicative of NTIS. Follow-up testing of abnormal TSH results was rarely performed. We recommend continued education of junior clinicians regarding thyroid function testing in critically ill patients.

Keywords. audit, cost-saving, non-thyroidal illness syndrome, thyroid function tests, thyroid-stimulating hormone.
\end{abstract}

S Afr J Crit Care 2020;36(1):46-50 https://doi.org/10.7196/SAJCC.2020.v36i1.406

\section{Contribution of the study}

This study describes the requesting pattern for TSH tests in patients admitted to the emergency department and intensive care unit at Tygerberg Hospital, South Africa. The findings emphasise the importance of prudent thyroid function testing in critically ill patients and follow-up in suspected cases of non-thyroidal illness syndrome.

Thyroid function testing is widely available and often requested in clinical scenarios when nonspecific signs and symptoms are present. Disorders unrelated to thyroid pathology, usually encountered in critically ill patients, can be accompanied by biochemical aberrancies. Abnormal thyroid profiles in critically ill patients are referred to either as non-thyroidal illness syndrome (NTIS) or as euthyroid sick syndrome (ESS). ${ }^{[1]}$ In day-to-day hospital practice, clinicians are regularly confronted with abnormal results from thyroid function tests. Differentiating NTIS or ESS from a pathological condition is essential yet challenging. ${ }^{[2]}$

The biochemical changes revealed by thyroid function testing during critical illness are not necessarily accompanied by clinical evidence of overt thyroid disease. The changes in thyroid biochemistry may mimic pathological pituitary insufficiency, yet in fact be an essential physiological adaptation of the thyroid hormone axis. The observed changes in thyroid function are attributed to altered secretion of hypothalamic thyrotropin-releasing hormone and thyroid-stimulating hormone (TSH), as well as to changes in protein binding, hormonal transport, variations in deiodinase enzyme activity or nuclear thyroid hormone receptor activity. ${ }^{[3]}$

Generally, thyroid function testing is not advised in the critically ill unless primary thyroid or pituitary pathology is clinically suspected. TSH measurement is the preferred screening test for thyroid disease, but TSH test results may be misleading and variable in critical illness. TSH evaluation in acutely ill patients without signs of overt thyrotoxicosis or hypothyroidism is therefore indicated only in select clinical scenarios, such as cardiac arrhythmias. ${ }^{[4]}$

Various non-thyroidal medical conditions may affect thyroid function results. The illnesses are usually of a substantial nature and may include systemic infections, inflammatory disorders and ischaemic conditions such as myocardial infarctions. ${ }^{[2]}$ Surgery, starvation and severe trauma may also lead to altered biochemical results in a thyroid function test. ${ }^{[2]}$ In NTIS, thyroid function is expected to normalise within 6 - 8 weeks of the main pathology resolving. ${ }^{[2]}$ To conclusively 
confirm NTIS, repeat biochemical testing with normalisation of thyroid function is essential. ${ }^{[5]}$

With the current cost of laboratory testing, clinical vigilance should prevail, especially in resource-constrained environments. At Tygerberg Hospital (TBH) in Cape Town, South Africa (SA), testing TSH levels is used as a screening test for thyroid abnormalities; if results are abnormal, further testing may be indicated. In critically ill patients, injudicious TSH testing may have considerable cost implications as additional investigations may be prompted unnecessarily. By eliminating inappropriate requests for TSH testing, the cost of pathology services and hospitalisation may be reduced.

This study describes the request pattern for TSH tests in patients admitted to the emergency department and intensive care units (ICUs) at TBH, Cape Town.

\section{Methods \\ Study setting and design}

This descriptive (observational) study was conducted in the Chemical Pathology Laboratory of the National Health Laboratory Services (NHLS), who provide diagnostic services to the public health sector at $\mathrm{TBH}$ and are accredited by the South African National Accreditation System. Tygerberg Hospital is affiliated with Stellenbosch University and functions as both a secondary and tertiary, multidisciplinary hospital. The hospital serves a population of 3.4 million people and has a bed capacity of 1384 , of which 164 are reserved for high-care and intensive-care purposes. The study retrospectively evaluated the pattern of requests for TSH tests in critically or acutely ill in-patients over a 6-month period (October 2016 - March 2017).

\section{Study population and study procedure}

All requests for TSH testing in patients older than 18 years who presented to the emergency department or were admitted to the medical, cardiac or surgical ICU or the medical high-care unit during the study period were included in the analysis. Patient data were collected from the NHLS laboratory information system (LabTrak). The information system was also used to determine whether cases of NTIS were followed up by repeat testing after 6 - 8 weeks. Clinical information required to determine the indication for TSH testing was obtained from the electronic patient record system of the hospital. Data collected included patient demographics, medication at index presentation and final clinical diagnosis.

Request records were excluded if the patient was younger than 18 years, pregnant, had known thyroid pathology, had a history of thyroid or parathyroid gland surgery, had a history of amiodarone or lithium therapy or if prior abnormal thyroid function results were documented on the laboratory information system.

\section{Laboratory measurements}

Serum TSH, free thyroxine $\left(\mathrm{fT}_{4}\right)$ and free triiodothyronine $\left(\mathrm{fT}_{3}\right)$ were measured on the Cobas e 601 analyser (Roche Diagnostics, Germany). TSH measurement involved a sandwich immunoassay followed by electrochemiluminescent detection. Measurement of $\mathrm{fT}_{4}$ and $\mathrm{fT}_{3}$ involved a competitive immunoassay followed by electrochemiluminescent detection.

Index TSH values were categorised as normal or abnormal. TSH levels were regarded as abnormal if outside the reference interval utilised at the NHLS (0.27 - $4.2 \mathrm{mIU} / \mathrm{L})$. Abnormal TSH results were then subcategorised as follows, based on whether active treatment was indicated:

- abnormal TSH for follow-up testing; no active treatment indicated

- abnormal TSH requiring active therapy.
The decision for treatment rested with the attending physician or surgeon.

In the case of an abnormal TSH result, it is current standard practice at the NHLS to perform an $\mathrm{fT}_{4}$ test by default. Even when the initial request is for comprehensive thyroid function testing, which includes $\mathrm{TSH}, \mathrm{fT}_{4}$ and $\mathrm{fT}_{3}$, the latter two will both be tested only if the TSH result is abnormal. Complete thyroid function testing at index presentation is permitted only in ICU settings and if motivated by the hospital's in-house endocrinologists.

\section{Statistical analysis}

Data were compiled using Microsoft Excel (Microsoft Corp., USA) and analysed using the STATA (version 15) statistical software package (Stata Corp., USA), in consultation with the Biostatistics Unit of the Faculty of Medicine and Health Sciences at Stellenbosch University.

The Shapiro-Wilk test was used to test for normally distributed data. Continuous variables were unevenly distributed and are reported as medians and interquartile ranges (IQRs).

\section{Ethical considerations}

The research complied with the Declaration of Helsinki - ethical principles for medical research involving human subjects - and the study was approved by the Ethical Review Board of the Faculty of Medicine and Health Sciences, Stellenbosch University (ref. no. S17/07/117). A waiver of informed consent applies to retrospective data collection.

\section{Results}

During the study period, 7912 and 2097 patients were admitted to the emergency department and ICU, respectively. A total of 5635 requests for TSH testing were received across all in-patient wards and outpatient clinics during the study period. A fifth of these requests ( $n=1$ 139/5 635) were from the emergency department and ICU combined. A TSH test was requested for one in every ten patients admitted to the emergency department ( $n=818 / 7912 ; 10 \%)$, and for one in every fourteen patients in ICU $(n=155 / 2$ 097; 7\%).

A total of 166 TSH tests from the emergency department and ICU were excluded owing to known thyroid pathology $(n=90)$, incomplete or absent clinical information $(n=55)$, pregnancy $(n=14)$ and interfering medication $(n=7)$. A total of 973 TSH test requests were consequently analysed (Fig. 1).

\section{Clinical presentation}

More than half of the patients for whom a TSH test was requested were female $(n=567 ; 58 \%)$, with a median (IQR) age of $53(37-67)$ years (Table 1). Most requests were from the Department of Medicine ( $n=807$; $83 \%$ ), and of these the majority were from the medical emergency division ( $n=757 ; 78 \%)$. Presenting complaints and assumed reasons for TSH testing were generally medical in nature and related to the neurological $(n=292 ; 30 \%)$, respiratory $(n=185 ; 19 \%)$, cardiovascular $(n=163 ; 17 \%)$ and gastrointestinal $(n=87 ; 9 \%)$ systems (Table 2$)$. In $25 \%$ of cases $(n=246)$, complaints were diverse and could not be linked to a specific system. In cases where the presenting complaint was specific, final diagnoses related mainly to either cardiovascular $(n=290 ; 30 \%)$ or neurological disease $(n=212 ; 22 \%)$ (Table 2$)$.

\section{Biochemical evaluation}

Requests for thyroid-stimulating hormone measurement

The median (IQR) concentration of TSH from the 973 tests evaluated was $1.69(0.98-2.91) \mathrm{mIU} / \mathrm{L}$ (Table 1). Abnormal levels were found 


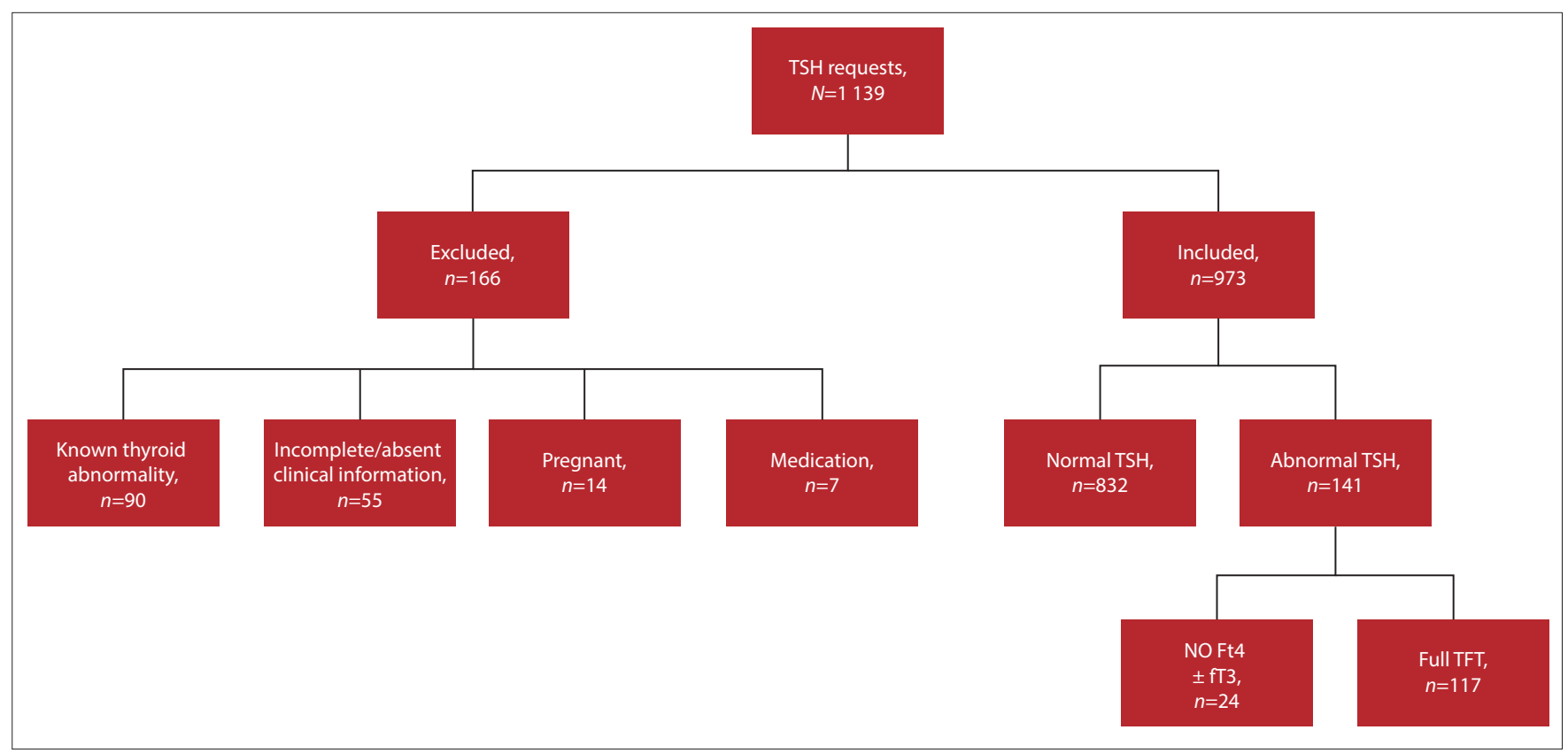

Fig. 1. Selection of study population. (TSH = thyroid-stimulating hormone; $T F T=$ thyroid function test; $f T_{4}=$ free thyroxine; $f T_{3}=$ free triiodothyronine).

in 141 cases $(14 \%)$. TSH levels were elevated in 113 of these cases $(80 \%)$. Low or suppressed TSH values were documented in the remaining $20 \%$ of the samples $(n=28)$. Approximately four out of five abnormal TSH results were attributed to NTIS or subclinical hypothyroidism ( $n=112 ; 79 \%)$. Results of follow-up TSH testing after 6 - 8 weeks were available in only $18 \%$ of the cases initially identified as abnormal $(n=25 / 141)$.

\section{Requests for comprehensive thyroid function testing}

As shown in Fig. 2, comprehensive thyroid function testing ( $\mathrm{TSH}$ and $\mathrm{fT}_{4}$ and/or $\mathrm{fT}_{3}$ ) was performed in 128 patients, prompted mostly by an abnormal TSH value ( $n=117 ; 91 \%) ; 11$ requests were received in the absence of any TSH abnormality. Among these latter requests, two patients had low $\mathrm{fT}_{4}$ and $\mathrm{fT}_{3}$ levels and one had a low $\mathrm{fT}_{4}$ level (no $\mathrm{fT}_{3}$ results available), indicative of possible central pathology. Euthyroidism was confirmed in eight cases based on normal TSH and circulating thyroid hormone levels. The majority of the comprehensive profiles indicated either NTIS or subclinical hypothyroidism ( $n=68 ; 53 \%)$. Subclinical hyperthyroidism was noted in 12 patients. In 29 patients with abnormal TSH results and in whom overt primary thyroid disease was suspected, the diagnosis was confirmed with more comprehensive testing, showing abnormal circulating levels of $\mathrm{fT}_{4}$. A biochemical profile in keeping with hypothyroidism was noted in 19 of these patients (66\%), whereas a thyroid hormone profile indicative of hyperthyroidism was noted in the remaining 10 patients

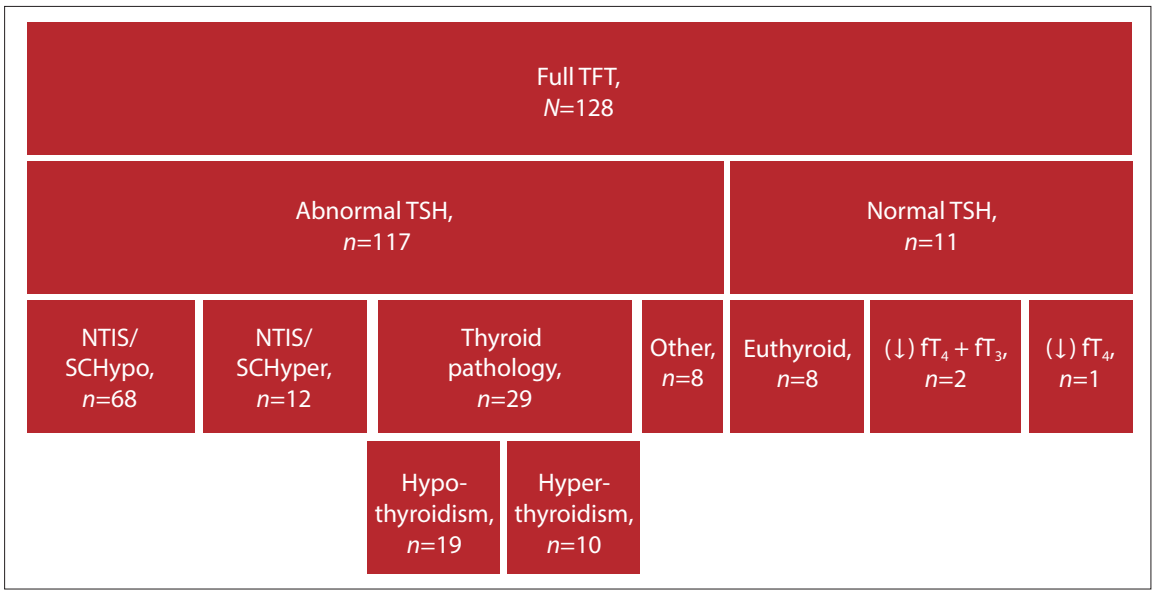

Fig. 2. Diagnostic interpretation of all full thyroid function tests $(\mathrm{N}=128)$. The majority of the profiles were suggestive of either non-thyroidal illness syndrome or subclinical hypothyroidism. (NTIS = non-thyroidal illness syndrome; SCHypo = subclinical hypothyroidism; SCHyper = subclinical hyperthyroidism; $\mathrm{TSH}=$ thyroid-stimulating hormone; TFT $=$ thyroid function test; $f T_{4}=$ free thyroxine; $f T_{3}=$ free triiodothyronine).

(34\%) (Table 3). Overt thyroid dysfunction was therefore biochemically confirmed in 29 of the 128 comprehensive thyroid function tests performed $(23 \%)$. In eight patients, the abnormal thyroid profile could be attributed to central hypothyroidism and medication such as steroids and dopamine.

In 24 of the 141 abnormal TSH results (17\%), $\mathrm{fT}_{4}$ or $\mathrm{fT}_{3}$ measurement was not performed, which conflicts with standard practice; this was found to be due to incorrect gatekeeping. Although gatekeeping can be reversed by clinicians, none on the blocked $\mathrm{fT}_{4}$ or $\mathrm{fT}_{3}$ tests was reinstalled.

\section{Overt primary hypothyroidism}

Overt primary hypothyroidism was noted in 19 patients (Table 3), based on elevated TSH levels (median (IQR) 8.33 (6.58 - 14.73) $\mathrm{mIU} / \mathrm{L}$ ) and low $\mathrm{fT}_{4}$ levels (median (IQR) 10.6 (8.9 - 11.9) $\mathrm{pmol} / \mathrm{L}$ ) $\mathrm{An} \mathrm{fT}_{3}$ value was available only in three cases. The main presenting symptoms in this subset of patients were related to the cardiovascular, respiratory and neurological systems. Cardiovascular symptoms were present in five patients and included angina $(n=4)$ and symptoms of congestive cardiac failure $(n=1)$. Dyspnoea and coughing attributed to the respiratory system were the main complaints in five patients and neurological features of confusion, headache, lower limb weakness, delirium and dizziness were evident in another four patients. The remaining five patients presented with 
Table 1. Descriptive data of all requests for TSH testing from ICUs and the ED during the study period

\begin{tabular}{llll}
\hline Variables & Total & Female & Male \\
\hline $\begin{array}{l}\text { TSH test } \\
\text { requests, } n(\%)\end{array}$ & 973 & $567(58.3)$ & $406(41.7)$ \\
$\begin{array}{l}\text { Age (years), } \\
\text { median (IQR) }\end{array}$ & $53(37-66)$ & $53(37-67)$ & $53(37-65)$ \\
$\begin{array}{l}\text { TSH (mIU/L), } \\
\text { median (IQR) }\end{array}$ & $1.69(1.03-2.9)$ & $1.73(0.98-2.91)$ & $1.65(1.09-2.89)$
\end{tabular}

$\mathrm{TSH}$ = thyroid-stimulating hormone; $\mathrm{IQR}=$ interquartile range; $\mathrm{ED}=$ emergency department; $\mathrm{IQR}$ = interquartile range.

Table 2. Categories of presenting complaints and final diagnosis

\begin{tabular}{lll}
\hline Diagnostic category & $\begin{array}{l}\text { Presenting } \\
\text { complaint, } \boldsymbol{n}(\%)\end{array}$ & $\begin{array}{l}\text { Final diagnosis, } \\
\boldsymbol{n}(\%)\end{array}$ \\
\hline Neurological & $292(30)$ & $212(22)$ \\
Respiratory & $185(19)$ & $75(8)$ \\
Cardiovascular & $163(17)$ & $290(30)$ \\
Gastrointestinal & $87(9)$ & $14(1)$ \\
Miscellaneous & $246(25)$ & $382(39)$
\end{tabular}

Table 3. Biochemical evaluation of comprehensive thyroid function testing according to referring department $(N=128)$

\begin{tabular}{llll}
\hline Biochemical evaluation & $\begin{array}{l}\text { Total, } \\
\boldsymbol{n}(\%)\end{array}$ & ED, $\boldsymbol{n}$ & ICU, $\boldsymbol{n}$ \\
\hline Abnormal TSH level & $117(91)$ & 90 & 27 \\
$\begin{array}{l}\text { NTIS/subclinical } \\
\text { hypothyroidism }\end{array}$ & $68(53)$ & 56 & 12 \\
$\begin{array}{l}\text { Subclinical } \\
\text { hyperthyroidism }\end{array}$ & $12(9)$ & 9 & 3 \\
$\begin{array}{l}\text { Primary hypothyroidism } \\
(N=29)\end{array}$ & $19(66)$ & 13 & 6 \\
$\begin{array}{l}\text { Primary hyperthyroidism } \\
(N=29)\end{array}$ & $10(34)$ & 10 & 0
\end{tabular}

$\mathrm{ED}=$ emergency department; $\mathrm{ICU}=$ intensive care unit;TSH = thyroid-stimulating hormone; NTIS = non-thyroidal illness syndrome.

miscellaneous complaints, which were not related to a specific organ system. The discharge diagnoses were of cardiovascular aetiology in 37\% of cases $(n=7)$.

\section{Overt primary hyperthyroidism}

In patients whose thyroid function profiles were compatible with primary hyperthyroidism $(n=10)$, TSH levels were consistently $<0.01 \mathrm{mIU} / \mathrm{L}$ and the median (IQR) fT 4 level was 50.9 (31.9 - 64.18) $\mathrm{pmol} / \mathrm{L}$. An $\mathrm{fT}_{3}$ measurement was performed in only two cases. Symptoms at presentation related mostly to the cardiovascular system ( $n=3$; chest pain, dyspnoea, palpitations) and the neurological system ( $n=3$; unilateral weakness, convulsion, headache). Most patients in this group $(n=8 ; 80 \%)$ were discharged with a clinical diagnosis of overt hyperthyroidism.

\section{Discussion}

TSH measurement was requested in a considerable number of patients admitted to the acute and critical-care services at Tygerberg Hospital during the study period. The majority of the TSH results were normal and most of the abnormal results were attributed to NTIS. A small number of patients $(n=29 / 973)$ were newly diagnosed with primary thyroid pathology (mostly hypothyroidism and with cardiovascularrelated symptoms) and it is concerning that a diagnosis of thyroid pathology was stated on the discharge notes in only $1 \%$ of patients ( $n=8 / 973)$ for whom a TSH test was requested. It is alarming that patients with abnormal TSH results were not followed up with repeat testing after resolution of the acute illness.

The general consensus is that thyroid function testing should not be requested in the critically ill, unless thyroid pathology is the suspected cause of or a notable contributor to the causative pathological condition. ${ }^{[4]}$ In our study, TSH testing was requested in $10 \%$ of patients considered to be acutely ill, five times more than what was reported by Giacomini et al. ${ }^{[4]}$ in an emergency department (1.9\%).

Emergency departments often display the shortcomings of the greater healthcare system they form part of. In SA, junior clinicians in emergency departments are often faced with a vast spectrum of diseases of varying severity in high-volume, resource-limited environments. Hypothyroidism can present with a variety of nonspecific complaints. Patients often present to the emergency department with nonspecific signs and symptoms and hypervigilance on the part of the clinician may contribute to regular thyroid testing. ${ }^{[6]}$

In their audits on thyroid function testing in acutely ill patients, Giacomini et al. ${ }^{[4]}$ and Lamb and Martin ${ }^{[5]}$ concluded that TSH measurement was inappropriately requested in $30 \%$ and $22 \%$ of cases, respectively. Thyroid pathology was diagnosed in $5 \%$ of cases in their studies. ${ }^{[4,5]}$ The lower occurrence of thyroid pathology in our study ( $3 \% ; n=29 / 973)$ may be due to differences in the severity of nonthyroidal illness, characteristics of the patients, background prevalence of thyroid disease, and lack of indicating thyroid pathology as part of the discharge diagnoses.

The majority of TSH results ( $n=832 ; 86 \%$ ) were normal in our study population. This is in keeping with the results reported by Lachman et al., ${ }^{[7]}$ who investigated the cost-effectiveness of routine thyroid function screening in adults at a psychiatric hospital during acute psychiatric presentation. They indicated that TSH results were normal in $79 \%$ of admissions.

In a similar study in the UK, Lamb and Martin ${ }^{[5]}$ reported that from a sample of 143 patients, $14 \%$ of abnormal TSH results were attributable to NTIS. The finding was based on a normal TSH level at follow-up, although a low rate of repeat testing limited generalisation. Likewise, only $18 \%$ of patients with abnormal TSH results received follow-up testing in our study, despite follow-up testing being recommended after 6 - 8 weeks. This limits our ability to categorise the diagnoses conclusively as NTIS. Failure to follow up abnormal results might be due to clinicians in the primary-care setting not being aware of the importance of a repeat TSH test, patients often being lost to follow-up once the acute illness has resolved, and NTIS not being stated on the discharge summary, which would flag the patient for a repeat TSH test. ${ }^{[8]}$

We found abnormal TSH results to be due mostly to elevated TSH levels. Giacomini et al. ${ }^{[4]}$ and Lamb and Martin ${ }^{[5]}$ reported a similar pattern in abnormal thyroid function first identified in the emergency department, and found a similar prevalence of primary hypothyroidism to that of our study ( $18 \%$ and $21 \%$, respectively). As $\mathrm{fT}_{3}$ measurements were not available in all cases of abnormal TSH results, we were unable to categorise all patients with elevated TSH levels.

Thyroid function testing is expensive: ZAR444.82 for a full thyroid function profile in the public sector (ZAR165.64 for TSH measurement, ZAR139.59 for testing $\mathrm{fT}_{4}$ and ZAR139.59 for testing $\mathrm{fT}_{3}$ ). In acutely ill patients, unnecessary requests for TSH testing have a notable cost implication. Abnormal TSH results will not only prompt testing of $\mathrm{fT}_{4}$ 
but also lead to follow-up testing 6 - 8 weeks after the acute illness has resolved. The total cost of such a scenario is ZAR470.87 (initial TSH testing plus $\mathrm{fT}_{4}$ plus repeat TSH). If the TSH test is requested only once the acute illness has resolved, results will most probably be normal, which translates to a cost saving of ZAR305.23 (a single TSH test at ZAR165.64, instead of the added $\mathrm{fT}_{4}$ and repeat TSH test). Inexperienced clinicians may also decide on imaging of the pituitary gland and even consider responding to the abnormal results with thyroid hormone replacement, with detrimental consequences. Lachman et al. ${ }^{[7]}$ concluded that TSH testing in acute psychiatric patients is not cost-effective, as screening will likely reveal temporary abnormalities that are not of significance. The results from our study support this conclusion in our emergency-care setting.

Although electronic gatekeeping as implemented by the NHLS has been shown to be an effective cost-saving tool, ${ }^{[9]}$ it was inappropriate in $17 \%$ of cases with abnormal TSH results. Rejected tests can be restored if a clinician contacts the laboratory. Interestingly, none of the rejected $\mathrm{fT}_{3}$ or $\mathrm{fT}_{4}$ requests in the current study was restored. Failure to restore these tests might indicate that TSH testing is routinely requested in the absence of a definite clinical indication and the results either were not reviewed or did not influence immediate patient management. ${ }^{[7,9]}$

\section{Study limitations}

Data were collected retrospectively from reviewing patient files, which may not always have been accurately or fully completed. We assumed patients presenting to the emergency department to be acutely or critically ill, but did not use the triage score to confirm this assumption. NTIS can also be associated with a TSH level within the normal range but a low $\mathrm{fT}_{3}$ level. As $\mathrm{fT}_{3}$ testing is not routinely performed in our laboratory when TSH results are normal, such NTIS cases could have been missed. Similarly, as $\mathrm{fT}_{4}$ is performed reflexively, inappropriately normal TSH results could have been missed. Nonetheless, central hypothyroidism is a rare condition, with an estimated prevalence of between 1 in 20000 and 1 in 80000 in the general population. ${ }^{[10]}$ The strength of our study, however, relates to both clinical and laboratory data being collected over a 6-month period. To the best of our knowledge, it is the first study of its kind in sSA.

\section{Conclusion}

TSH testing is commonly requested in the emergency-care setting, with the majority of results being normal. Although thyroid pathology can manifest with nonspecific symptoms and so prompt frequent requests for TSH testing, it was found to have a low prevalence in our setting, similar to findings from other centres. If TSH is abnormal during initial presentation and NTIS is suspected, follow-up is essential. We found that follow-up testing of abnormal TSH results was rarely performed, and we therefore recommend that such patients be flagged for follow-up at discharge.

Declaration. None.

Acknowledgements. None.

Author contributions. ECK conceptualised the study, collected the data and wrote the manuscript. MC, AC and MH contributed to assessment of the findings and proofreading the article prior to submission.

Funding. None.

Conflicts of interest. None.

1. McIver B, Gorman CA. Euthyroid sick syndrome: An overview. Thyroid 1997;7(1):125-132. https://doi.org/10.1089/thy.1997.7.125

2. Fliers E, Bianco AC, Langouche L, Boelen A. Thyroid function in critically ill patients. Lancet Diabetes Endocrinol 2015;3(10):816-825. https://doi.org/10.1016/s2213-8587(15)00225-9

3. Farwell AP. Nonthyroidal illness syndrome. Curr Opin Endocrinol Diabetes Obes 2013;20(5):478 484. https://doi.org/10.1097/01.med.0000433069.09294.e8

4. Giacomini A, Chiesa M, Carraro P. Urgent thyroid-stimulating hormone testing in emergency medicine: A useful tool? J Emerg Med 2015;49(4):481-487. https://doi.org/10.1016/j. emermed.2015.05.003

5. Lamb EJ, Martin J. Thyroid function tests: Often justied in the acutely ill. Ann Clin Biochem 2000;37:158-164. https://doi.org/10.1258/0004563001899159

6. Chen YJ, Hou SK, How CK, et al. Diagnosis of unrecognized primary overt hypothyroidism in the ED. Am J Emerg Med 2010;28(8):866-870. https://doi.org/10.1016/j.ajem.2009.04.024

7. Lachman A, Cloete K, Kidd M, Schoeman R. The clinical utility and cost effectiveness of routine thyroid screening in adult psychiatric patients presenting at Stikland Hospital, Cape Town, South thyroid screening in adult psychiatric patients presenting at Stikland Hospital, Cape
Africa. Afr J Psychiatry 2012;15(1):36-41. https://doi.org/10.4314/ajpsy.v15i1.5

8. Premawardhana LD. Thyroid testing in acutely ill patients may be an expensive distraction. Biochem Med 2017;27(2):300-307. https://doi.org/10.11613\%2FBM.2017.033

9. Smit I, Zemlin AE, Erasmus RT. Demand management: An audit of chemical pathology test Biochem 2015;52(Pt 4):481-487. https://doi.org/10.1177/0004563214567688

10. Persani L. Central hypothyroidism: Pathogenic, diagnostic and therapeutic challenges. J Clin Endocrinol Metab 2012;97(9):3068-3078. https://doi.org/10.1210/jc.2012-1616 